\title{
Research on Steady Voltage Control Circuit of Small Wind Driven Generator
}

\author{
Anmin Wang ${ }^{1, a}$, Zhipeng Zhao ${ }^{1, b^{*}}$, Xiaojun Wang ${ }^{1, c}$ and Shujia $\mathrm{Li}^{1, \mathrm{~d}}$ \\ ${ }^{1}$ Qingdao University of Science \& Technology, Shandong, China \\ a1049775677@qq.com, b760907040@qq.com, '992007179@qq.com, d601627764@qq.com \\ *The corresponding author
}

Keywords: Wind power generation; Steady voltage; Buck chopping; UC3842

\begin{abstract}
The output voltage of small wind driven generator presented non-cyclical fluctuation affected by wind speed, which then leads to unstable accumulator charging voltage. In order to improve charging efficiency and prolong accumulator's service life, this paper designed a steady voltage circuit based on UC3842. It is mainly composed by rectifier filter circuit, Buck chopper circuit and post-processing circuit. With wind power variation, the duty ratio of PWM wave outputted by UC3842 is changing accordingly. Then, the circuit will output steady voltage to charge accumulators. This design is characterized by simple circuit, relatively low cost and convenient maintenance etc.
\end{abstract}

\section{Introduction}

The electric-generation principle of small wind driven generators: the impeller drives rotation of principal axis and then the generator output voltage charges accumulators [1]. With variation of wind speed, the rotation speed of impeller will be changing accordingly. Then, the output voltage will show non-cyclical fluctuation. The accumulator cannot be charged safely without steady voltage control circuit to guarantee voltage stability. At present, the steady voltage control circuit of small wind driven generators is characterized by complex circuit, inconvenient maintenance and relatively high cost etc, which restricts development of wind power industry.

This paper simplifies circuit configuration and decreases cost of steady voltage control circuit of wind power generation so as to provide convenience for post-maintenance based on steady voltage. The steady voltage control circuit designs in this paper mainly adopted Buck chopper circuit and power management chip UC3842 to achieve steady voltage.

\section{Working Principle of Steady Voltage Circuit}

Fig. 1 refers to designed working principle of steady voltage control circuit. The wind driven generator outputs three-phase AC, obtains DC with relatively small pulsating quantity after regulating rectifier filter circuit and then gets through MOS tube as Buck chopper circuit of switch power tub as well as stable DC after outputting filter circuit [2-3]. Besides, the feedback circuit composed by three-phase steady voltage reference source TL431 and coupling EL817 conducts sampling on accumulator charging voltage; the feedback signal enters into UC3842 and the duty ratio[4-5] of PWM wave is adjusted after regulating and comparison inside chip. The PWM wave transmits by chips controls MOS tube break-over and turn-off time so as to stabilize output voltage via closed-loop circuit.

\section{Hardware Design}

Selection of Accumulator and Generator. This paper selects common lead-acid battery with $24 \mathrm{~V}$ voltage and $48 \mathrm{AH}$ capacity in the market. The constant voltage charge is adopted with charging voltage of $28 \mathrm{~V}$. 


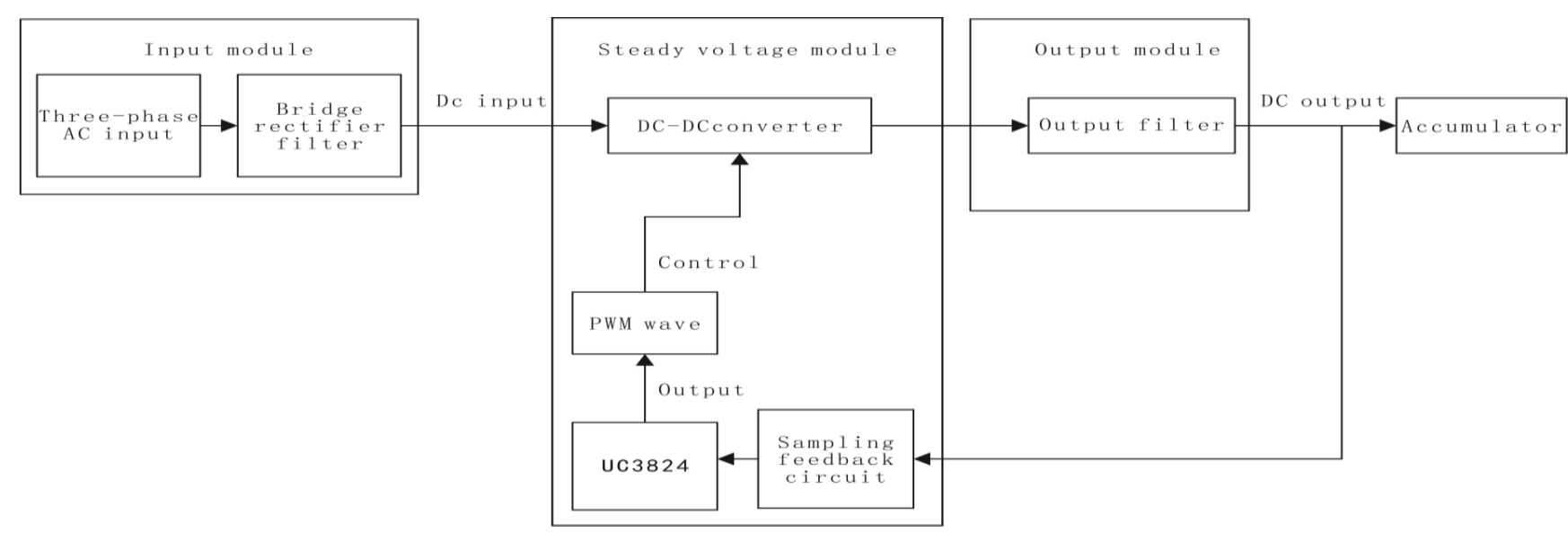

Figure 1. Block diagram of steady voltage circuit

This paper adopts permanent-magnetic direct-drive generator in the market that can represents most of products. The model is TYD250-300. The specific parameters are shown in Table 1. It is found through actual measurement that the output voltage of generator under normal operation is between $30 \mathrm{~V}$ to $85 \mathrm{~V}$. When the output voltage is lower than $28 \mathrm{~V}$, the rotating speed and output power will be lower than 10 prm and $35 \mathrm{~W}$ respectively. If the low voltage outputted by generator is boosted to charge accumulator, then the output current is very small with rather complex circuit and high production maintenance cost. Hence, this paper only designs depressurizing system.

Table 1 Electrical specification of permanent-magnetic direct-drive generator

\begin{tabular}{|c|c|}
\hline \multicolumn{2}{|c|}{ Electrical specification } \\
\hline Rated output power [W] & 300 \\
\hline No-load voltage [V] & 81 \\
\hline Nominal voltage [V] & 61 \\
\hline Rated current [A] & 5.1 \\
\hline Output & Three-phase AC \\
\hline
\end{tabular}

Introduction to Power Management Chip. The main circuit of steady voltage circuit in this paper adopts power management chip UC3842. This chip with fixes working frequency can output PWM wave with adjustable pulse length. It has totally 8 pins with the following functions: pin 1 is output terminal of error amplifier; pin 2 is input terminal of feedback voltage; pin 3 is input terminal of current detection; when detection voltage exceeded $1 \mathrm{~V}$, the chip will stop to output PWM; pin 4 is timing terminal; the working frequency of internal oscillator is determined by external resistance-capacitance; pin 5 is ground terminal; pin 6 is PWM output terminal; pin 7 is DC supply terminal of $16 \mathrm{~V}$ starting voltage with minimum and maximum supply voltage of $10 \mathrm{~V}$ and $30 \mathrm{~V}$ respectively. The chip will stop works with voltage lower than $10 \mathrm{~V}$; pin 8 is output terminal of $5 \mathrm{~V}$ reference voltage [6-7]. In this paper, pin 2 of UC3842 is connected to ground; pin 1 is taken as feedback one directly by skipping over amplifier inside UC3842hic, which saves feedback time. The chip UC3842 as well as its peripheral circuit is shown in Fig. 2.

Input Module Design. The steady voltage control circuit of wind driven generator mainly included input module, steady voltage module and output module [8]. The input module includes rectifier circuit and filter circuit. This design achieves conversion from AC to DC through rectifier filter circuit eventually [9].

Steady Voltage Module Design. The DC voltage obtained via rectifier filter circuit is unstable due to large output voltage fluctuation. The voltage should be regulated via steady voltage module. The steady voltage module includes UC3842 as well as its peripheral circuit, DC-DC converter and sampling feedback circuit, of which UC3842 as well as its peripheral circuit has been mentioned as above. 


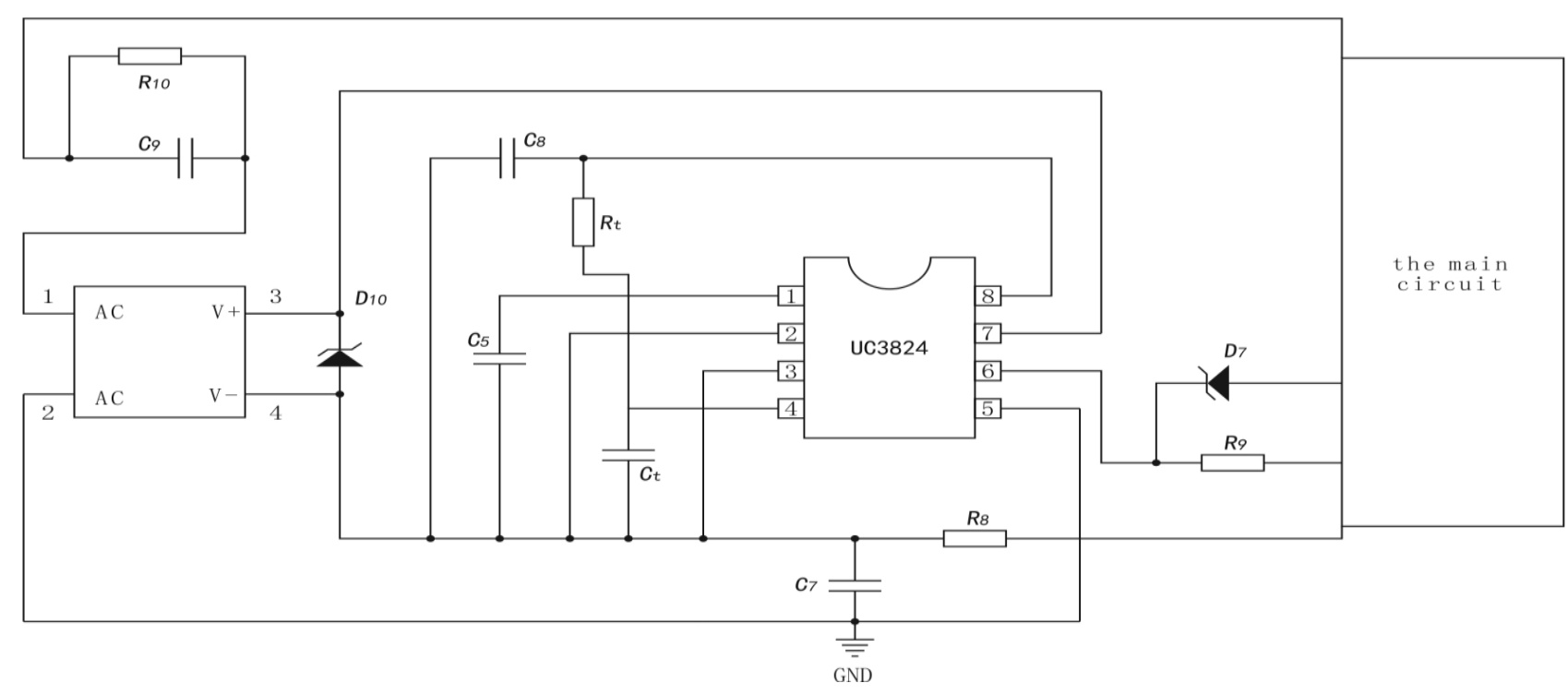

Figure 2. UC3842 as well as its peripheral circuit

(1) Design of DC-DC converter

The design of DC-DC converter includes the MOSFET, electrical inductor L1 and fly-wheel diode [10]. Its circuit diagram is shown in Fig. 3. The working principle of DC-DC converter: when MOSFET gets through, the output voltage of input module can charge accumulators through energy storage inductor L1, in which inductance obtains partial energy. The subsequent energy storage capacitor $\mathrm{C} 3$ also storages a part of energy; after a certain time, the MOSFET is disconnected, the inductor L1 will continue to charge accumulators. At this time, the diode D8 functions as follow current to consist of current loop. The divider resistance R6 will detect the output voltage and then feedback to control circuit, then the control circuit will change duty ratio of PWM wave, MOSFET closing and turn-off time, which can control output voltage and achieve steady voltage.

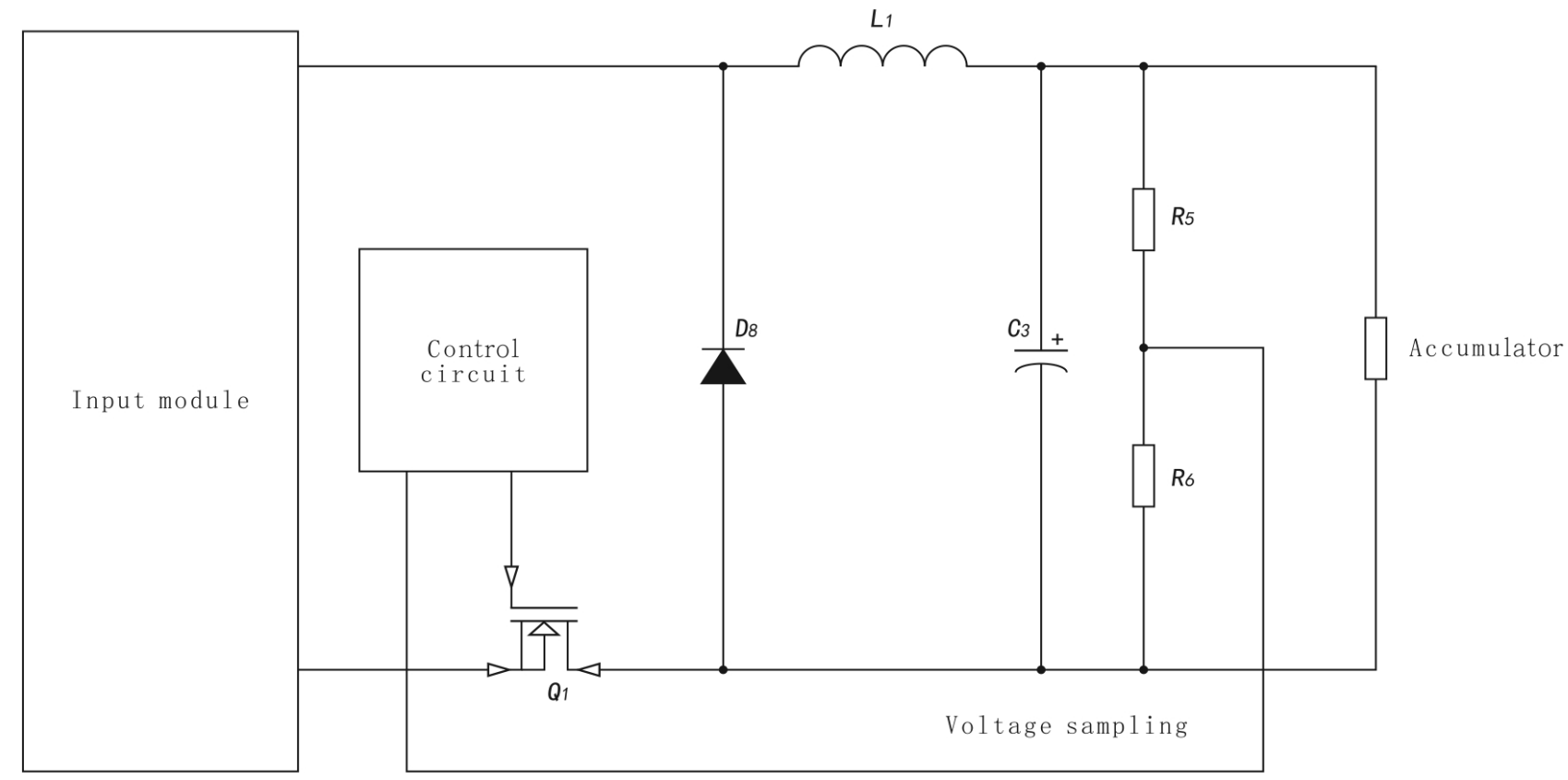

Figure 3. Circuit diagram of DC-DC converter

(2) Design of sampling feedback circuit

The output voltage is separated through R5 and R6. The voltage on R6 is sampling voltage. The three-terminal controllable reference source TL431 is used to obtain sampling voltage. The TL431 will change liner coupling El817's break-over degree by comparing sampling voltage and $2.5 \mathrm{v}$ 
reference voltage. Such changes will affect electric potential of UC3842 pit 1 so as to affect duty ratio of UC3842 output PWM wave. The diagram of sampling feedback circuit is shown in Fig.4.

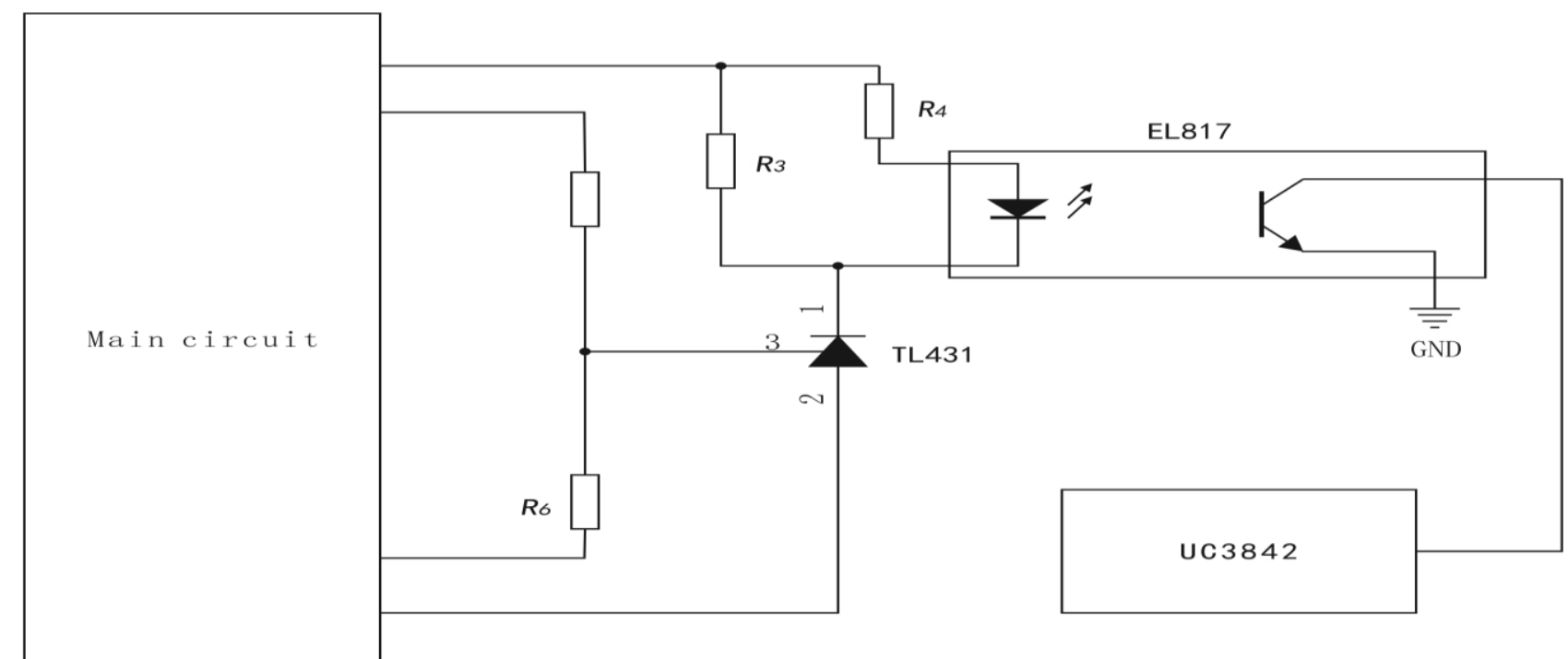

Figure 4. Diagram of sampling feedback circuit

Design of output Module. The DC voltage after processing via DC-DC converter still has highfrequency and low-frequency clutters. In order to guarantee smooth voltage of accumulator charging, the ceramic capacitor $\mathrm{C} 4$ and electrolytic capacitor $\mathrm{C} 3$ are used to conduct filtering on output voltage, of which electrolytic capacitor C3 also functions as energy storage in this design.

\section{Overall Circuit Diagram and Circuit Board}

The overall circuit diagram of steady voltage circuit designed in this paper is shown in Fig. 5.

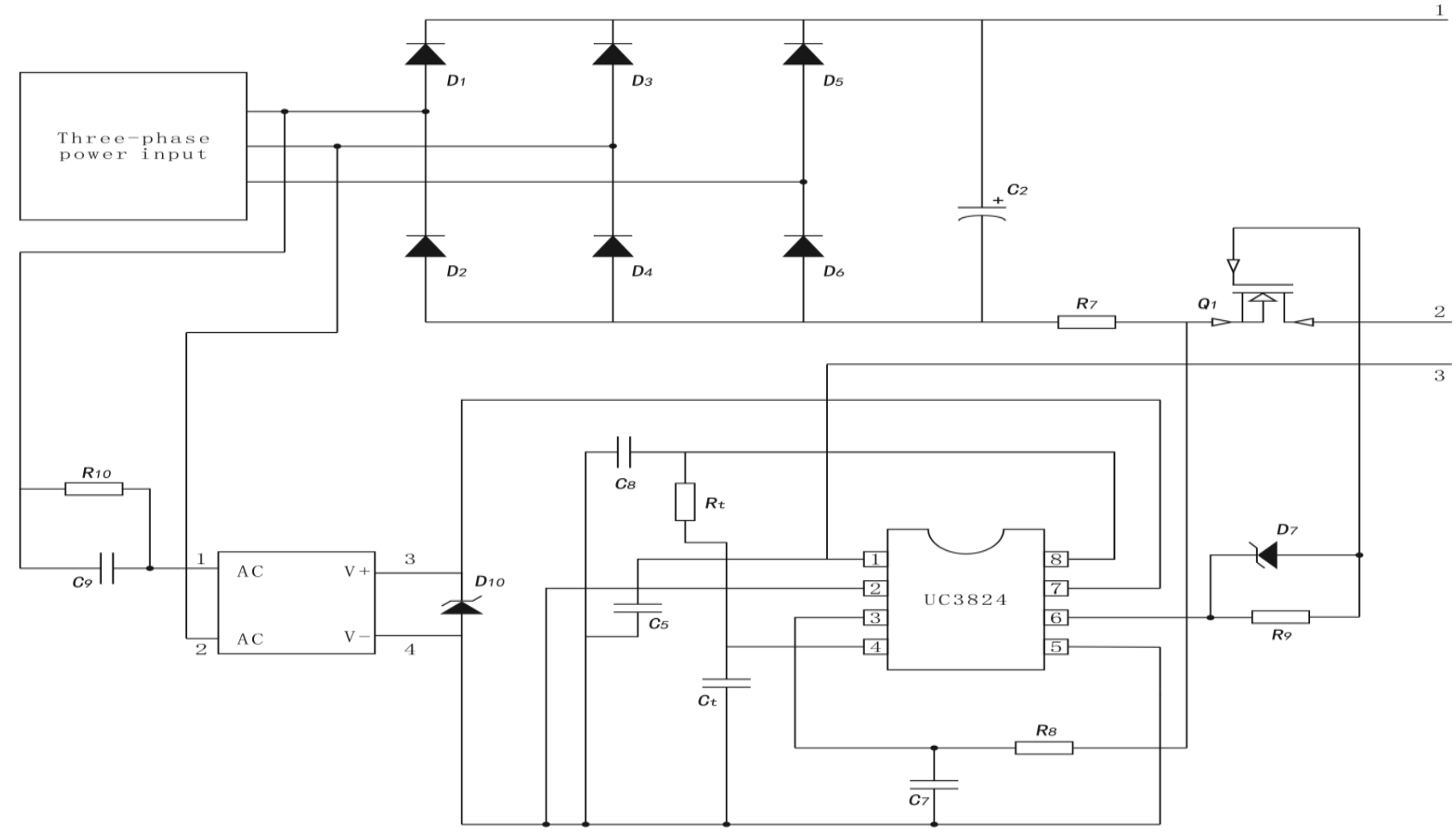




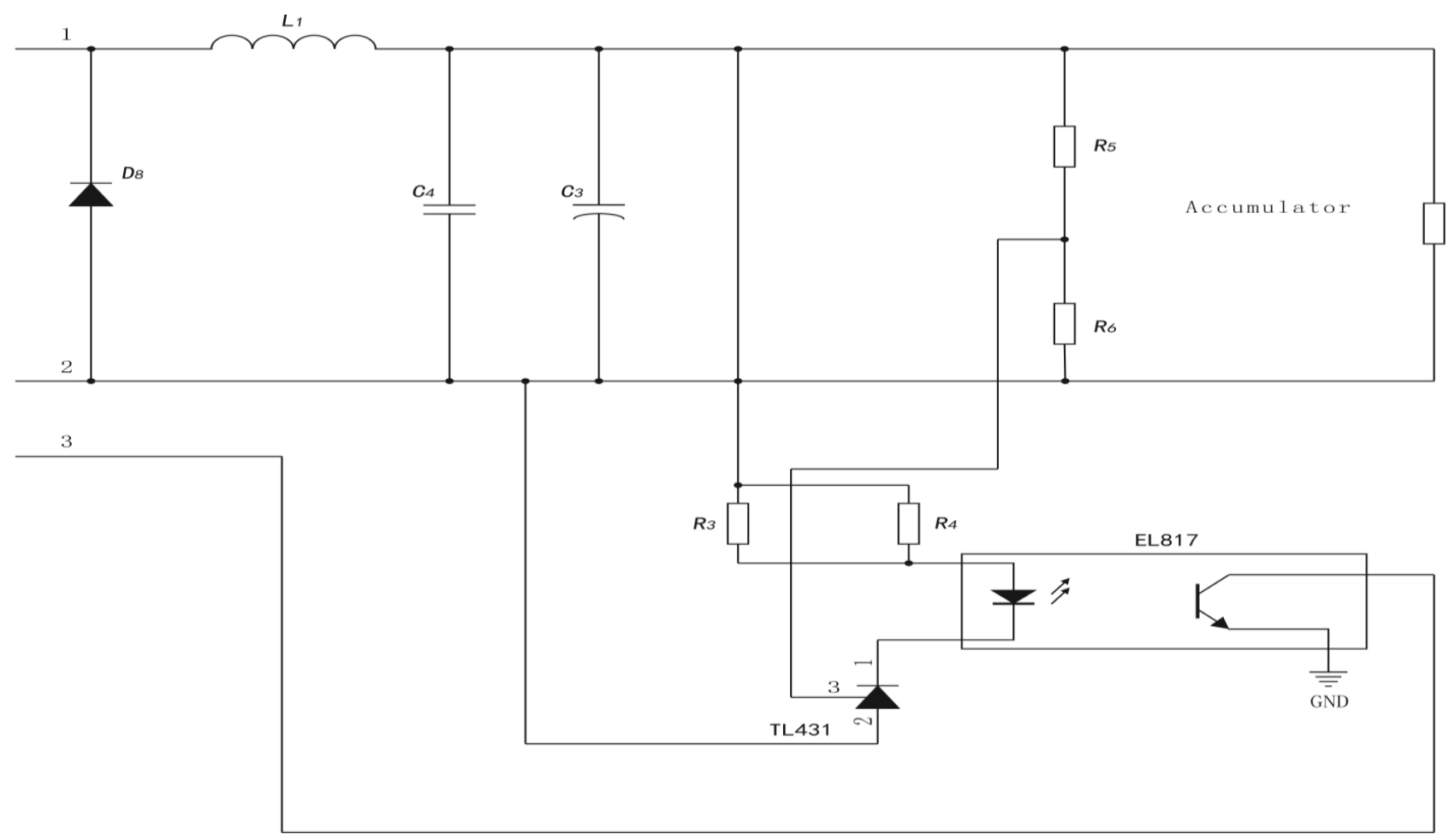

Figure 5. Circuit diagram of steady voltage circuit

\section{Experimental Results}

When the input voltage is 50V, duty ratio of UC3842 pit 6 is shown in Fig. 6.

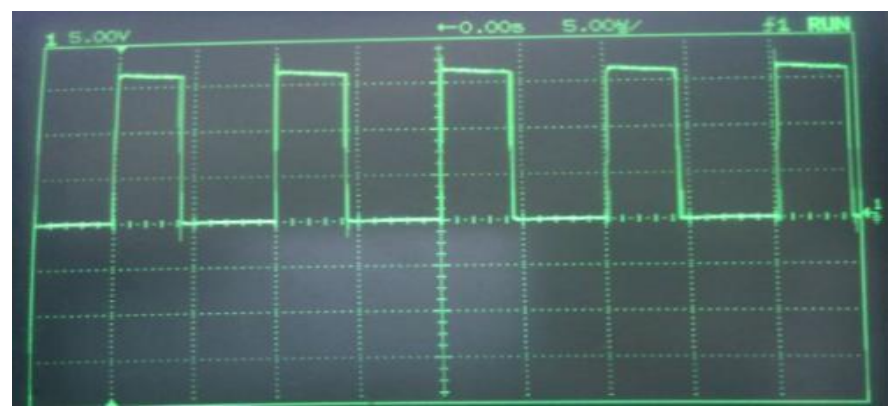

Figure 6. PWM waveform

The main spindle speed of generator varies with the changing output voltage. The output voltage of steady voltage circuit in this paper remains $28 \mathrm{~V}$. The output voltage waveform is shown in Fig. 7. The dead center position refers to the first dotted line from bottom to up. One grid in the figure represents $5 \mathrm{~V}$.

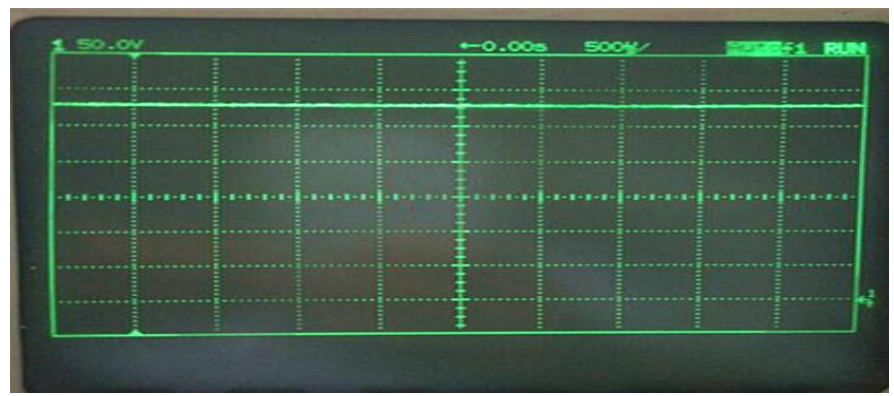

Figure 7. Output voltage diagram of steady voltage circuit 


\section{Conclusion}

This paper takes advantage of steady voltage control circuit of wind power generation designed by Buck chopper circuit and power management chip UC3842 to solve unstable output voltage of small wind driven generators and abnormal accumulator charging. It is characterized by simple structure, cheap cost and good maintainability etc.

\section{References}

[1] Shanquan Niu: Wind technology (Science Press, Beijing 2005).(In Chinese)

[2] Dingxuan Li: Design and application of switching stabilized power supply (Electric Power Press, Beijing 2006). (In Chinese)

[3] Yaofu Heng, Fujun Chen: Electric Power Automation Equipment. Vol. 29. (2009) No.9, p.133. (In Chinese)

[4] Shunli Liu: Practical technology of modern high-frequency switching power supply (Electronic Industry Press, Beijing 2001). (In Chinese)

[5] Yongyi Xue, Shuying and Wang and Xicai He: Application example of new-type power circuit (Electronic Industry Press, Beijing 2001). (In Chinese)

[6] Enxuan Hui: Electronic and Automation, (2000) No.9, p.45. (In Chinese)

[7] Juncheng Hu: Instrument Technology, (2005) No.3, p.79. (In Chinese)

[8] Liqiang $\mathrm{Hu}$, Zhenghui Lin and Linshen Li: Microelectronics, Vol. 33.(2003) No.2, p.136. (In Chinese)

[9] Xinge Wei, Shensheng Li: Basis of analog electronics technique (Electronic Industry Press, Beijing 2005). (In Chinese)

[10] Zhimin Zhou, Aihua Ji: Zero-based switching power supply design. Basics (Electronic Industry Press, Beijing 2013). (In Chinese) 